\title{
An Adaptive Non-linear Statistical Salt-and-Pepper Noise Removal Algorithm using Interquartile Range
}

\author{
Amiya Halder $^{a}$, Pritam Bhattacharya ${ }^{a}$, and Apurba Sarkar ${ }^{b}$ \\ ${ }^{a}$ St. Thomas College of Engineering and Technology, 4. D. H. Road, Kolkata -700023, India \\ ${ }^{b}$ Department of Computer Science and Technology, IIEST Shibpuer, Howrah -711103, India
}

Received 29th of August 2017; accepted 30th march 2019

\begin{abstract}
This paper presents a salt-and-pepper noise removal scheme using modified mean filter. The proposed method is based on a simple basic concepts of mean filter, where each mean value is calculated from the mathematical formula of interquartile range (IQR). It replaces the noisy pixels using IQR based mathematical formula applied on the filter window. Experimental results are presented to demonstrate the efficiency (quality of the image) of the method compared to other existing different types of impulse noise removal techniques.
\end{abstract}

Key Words: Salt-and-pepper noise, Median filter, IQR, PSNR.

\section{Introduction}

Salt-and-Pepper noise is a sparsely present white and black pixels as a result of sudden change of maximum (255) and minimum intensity (0) values of pixels. These type of noises generally get introduced in the image during image acquisition phase due to noisy sensor and bit errors in transmission. Salt-and-Pepper noise creates problem in communication for transmission of digital images. These noises needed to be eliminated before the image is fed as input to different image processing applications such as image enhancement, segmentation and recognition etc.

Researchers have been working in this area for decades and various algorithms for removing salt and pepper noise have been proposed. Every algorithm proposed in the literature has its own advantages and disadvantages. It has been observed that, most of the existing noise removal algorithms in the literature are based on linear (such as average filter) and nonlinear (such as standard median filter and modified median filter). Simplest among them is standard median filter (SMF) [1]. It takes a local window of intensities and finds out the median of the intensities which replaces the central pixel of given window in the image. This is highly used due to its simplicity and effectiveness, but this algorithm fails to produce satisfactory results if the noise percentage is more than $40 \%$ causing a high blurring effect in the image.

Correspondence to: < amiya.halder77@gmail.com>

Recommended for acceptance by <Angel Sappa >

https://doi.org/10.5565/rev/elcvia.1145

ELCVIA ISSN:1577-5097

Published by Computer Vision Center / Universitat Autonoma de Barcelona, Barcelona, Spain 
In the case of weighted median filter (WMF), the standard median filter is modified by assigning the weight value to some of the pixels within a window and in the case of center weighted median filter (CWMF), maximum weight value is set to the central pixel in a window [3,4]. These weights control the smoothing behavior of the image. WMF, CWMF and other adaptive median filters preserve fine details at the low level of noise density. The tristate median filter (TSMF), which is combination of SMF and CWMF filter is proposed in [5] is able to preserve the fine details but can not work well in case the image has high density of salt-and-pepper noise.

To improve the limitation of median based filters, different types of fuzzy filters and switching median filters have been proposed [10, 11, 13, 12]. In [6], Luo has proposed an efficient technique to remove impulse noise using fuzzy impulse detection(FIDT) technique. The technique presented therein can remove salt-and-pepper noise very efficiently even from a corrupted noise while preserving the image details. Also, in the year 2008, an efficient edge preserving technique has been developed which reduce impulse noise without degrading the image's finer details [9]. These methods work well for images having up to 50\% impulse noise density.

To remove the high density of impulse noise and preserve the fine details of image, different kinds of techniques have been proposed, like the noise adaptive fuzzy switching median filter (AFSMF) [15], modified decision based unsymmetric trimmed median filter (UTMF) [16], the the fast and efficient median filter (FEMF) [14], sorted switching median filter [17], decision-based coupled window median filter [18], improvement of Decision median filter for suppression of salt-and-pepper noise (IDMF)[2], new decision-based trimmed median filter (DTMF) [19] and an efficient restoration algorithm [ERA] [20] etc.

In this paper, we present a new type of modified mean filter for detecting and removing impulse noise using mathematical concept of median and interquartile range (IQR). The proposed filtering technique is simple and adaptive. The adaptive nature allows the filter to increase the size of its window according to local noise density.

Rest of the paper is organized as follows. The proposed method is introduced in Sec. 2. Section 3 presents simulation results obtained by the proposed method with brief analysis and provides comparison with existing techniques. Finally, in Sec. 4 the paper is concluded.

\section{Proposed Method}

The proposed filter is designed by modifying the existing adaptive modified mean filter with mathematical concepts of the Nonparametric Univariate Statistics for Quantitative Variables: the median and the interquartile range (IQR). To explain, let us consider $\xi(P, Q)$ be an image with the number of rows as $P$ and number of columns as $Q$. The method processes the noisy images by detecting the salt-and-pepper noise. Salt noises are the pixels with intensity value 0 and pepper noises are the pixels with intensity values 255 . If the intensity of the pixel being processed lies between 0 and 255 then it is a noise free pixel and it is left unchanged. On the other hand if the intensity value of the pixel is equal to 0 or 255 , then it is a noisy pixel and is processed by proposed filter as follows. If a noisy pixel is found then a square filtering window $H_{(2 w+1)(2 w+1)}(\mu, \nu)=$ $\{h(\mu+m, \nu+n)\}$, where $h(\mu, \nu)$ is pixel intensity of point $(\mu, \nu)$ and $m, n \in(-w, \ldots . ., \ldots w)$ is considered. From this window, non-noisy pixels are found out. If all the pixels are noisy, then the window size is increased. Let $N_{\mu, \nu}$ is defined as the set of all non-noisy pixels in the neighborhood of the point $h(\mu, \nu)$.

$$
N_{\mu, \nu}=\{h(k, l) \neq(0,255): k=[\mu-m, \mu+m], l=[\nu-n, \nu+n]\}
$$

Let the members of the set $N_{\mu, \nu}$ are $Z_{p+1}, Z_{p+2}, Z_{p+3} \ldots, Z_{p+q}$ where $q$ is the number of elements in $N_{\mu, \nu}$. These noise free pixels are arranged in the sorted order of intensity value i.e., $Z_{p+1}<=Z_{p+2}<=\ldots$. $<=$ $Z_{p+q}$. Then the index of the minimum value $\left(\chi_{l}\right)$ and the maximum value $\left(\chi_{u}\right)$ in sorted list are selected. The interquartile range (IQR) is the range of values within which reside in the middle $50 \%$ of the elements with the middle element at $\frac{\left(\chi_{l}+\chi_{u}\right)}{2}$. Now, if we consider total number elements which is $\left(\chi_{u}-\chi_{l}+1\right)$, then range of the IQR is from index $\frac{\left(\chi_{l}+\chi_{u}\right)}{4}$ to index $\frac{3 *\left(\chi_{l}+\chi_{u}\right)}{4}$. The lower bound of the interquartile range is called the first quartile $\left(P_{1}\right)$ of the list and contains all the elements in the index range from $\chi_{l}$ to $\frac{\left(\chi_{l}+\chi_{u}\right)}{4}$. The upper bound 
of the interquartile range is called the third quartile $\left(P_{3}\right)$ of the list and contains all the elements in the index ranges from $\frac{3 *\left(\chi_{l}+\chi_{u}\right)}{4}$ to $\left(\chi_{u}-\chi_{l}+1\right)$.

The proposed method is based on the above concepts. In the case of average filter, the center pixel is replaced by the average value of the $(2 w+1) \times(2 w+1)$ window. But, in the proposed method following formula is used.

$$
S_{\mu, \nu}=\frac{\sum_{q=\frac{\left(\chi_{l}+\chi_{u}\right)}{4}}^{\frac{3 *\left(\chi_{l}+\chi_{u}\right)}{4}} Z_{q}}{\frac{3 *\left(\chi_{l}+\chi_{u}\right)}{4}-\frac{\left(\chi_{l}+\chi_{u}\right)}{4}+1}
$$

In the case of median filter all non noisy pixels in the $(2 w+1) \times(2 w+1)$ window are sorted in the ascending order of their intensity value, and the median of the sorted values are found out. Subsequently, the noisy pixels are replaced with this median. But in that case the precision of the noise removal is very poor. It is observed that, the values within the range $\frac{\left(\chi_{l}+\chi_{u}\right)}{4}$ to $\frac{3 *\left(\chi_{l}+\chi_{u}\right)}{4}$ are near to median and far to 0 and 255 . So, in the proposed method, the noisy pixels are replaced with the mean of the range from $\frac{\left(\chi_{l}+\chi_{u}\right)}{4}$ to $\frac{3 *\left(\chi_{l}+\chi_{u}\right)}{4}$ producing a better precision. It is to be noted here that we are not considering the range $\chi_{l}$ to $\frac{\left(\chi_{l}+\chi_{u}\right)}{4}$ because they will be blackish in nature and also not considering the range $\frac{3 *\left(\chi_{l}+\chi_{u}\right)}{4}$ to $\left(\chi_{u}-\chi_{l}+1\right)$ because they will be whitish in nature.

\subsection{Explanation of the proposed method}

The working principle of the proposed method is described using a $3 \times 3$ selected window as follows:

\begin{tabular}{|l|l|l|}
\hline 0 & 20 & 40 \\
\hline 50 & 255 & 0 \\
\hline 132 & 0 & 90 \\
\hline
\end{tabular}

Let us consider the noisy pixel is at the center of the selected window 255 (processing pixel) and neighbours of this pixels are $N=\{20,40,50,132,90\}$ (noise free pixels). Now, these values are sorted in ascending order $N=\{20,40,50,90,132\}$ and the total number of pixel is $n=5$, the lower index is $\chi_{l}=0$, upper index is $\chi_{u}=4$ and median of the indices is $\frac{\left(\chi_{l}+\chi_{u}\right)}{2}=2$. So, IQR is calculated from $1(n / 4=5 / 4=1)$ to $3(3 n / 4=3 * 5 / 4=3)$, and its value is

$$
S_{\mu, \nu}=\frac{\sum_{q=\frac{5}{4}}^{\frac{3 * 5}{4}} Z_{q}}{\frac{3 * 5}{4}-\frac{5}{4}+1}=\frac{\sum_{q=1}^{3} Z_{q}}{3-1+1}=\frac{\sum_{q=1}^{3} Z_{q}}{3}=\frac{Z_{1}+Z_{2}+Z_{3}}{3}=\frac{40+50+90}{3}=60
$$

Finally, the noisy pixel 255 (center of the window) is replaced by the value of 60 (i.e. $S_{\mu, \nu}$ ). After removing the noisy pixel, the modified selected window is shown below:

\begin{tabular}{|l|l|l|}
\hline 0 & 20 & 40 \\
\hline 50 & 60 & 0 \\
\hline 132 & 0 & 90 \\
\hline
\end{tabular}

In case the center pixel in the selected $3 \times 3$ window is noisy ( 0 or 255 ) and all neighborhood pixels of window are also noisy, then the window size is increased to $5 \times 5,7 \times 7, \ldots$ and so on. The process is explained again with a window size of $5 \times 5$.

The center is 0 of the $3 \times 3$ selected window and all the neighbors are also noisy pixels, so increased the window size $5 \times 5$. In $5 \times 5$ window, the noise free pixels are $N=\{60,20,40,30,50,45,70,80,55\}$. Now, these values are sorted in ascending order $N=\{20,30,40,45,50,55,60,70,80\}$ and the total number of pixel 


\begin{tabular}{|l|l|l|l|l|}
\hline 60 & 20 & 40 & 30 & 255 \\
\hline 50 & 0 & 0 & 255 & 45 \\
\hline 70 & 0 & 0 & 0 & 255 \\
\hline 80 & 0 & 0 & 0 & 255 \\
\hline 255 & 55 & 0 & 0 & 0 \\
\hline
\end{tabular}

is $n=9$, the lower index is $\chi_{l}=0$, upper index is $\chi_{u}=8$ and median of the indices is $\frac{\left(\chi_{l}+\chi_{u}\right)}{2}=4$. So, IQR is calculated from $2(n / 4=9 / 4=2)$ to $6(3 n / 4=3 * 9 / 4=6)$, and its value is

$S_{\mu, \nu}=\frac{\sum_{q=\frac{9}{4}}^{\frac{3 * 9}{4}} Z_{q}}{\frac{3 * 9}{4}-\frac{9}{4}+1}=\frac{\sum_{q=2}^{6} Z_{q}}{6-2+1}=\frac{\sum_{q=2}^{6} Z_{q}}{5}=\frac{Z_{2}+Z_{3}+Z_{4}+Z_{5}+Z_{6}}{5}=\frac{40+45+50+55+60}{5}=50$

The noisy pixel 0 (center of the window) is replaced by the value of 50 (i.e. $S_{\mu, \nu}$ ). After removing the noisy pixel, the processing window is shown below:

\begin{tabular}{|l|l|l|l|l|}
\hline 60 & 20 & 40 & 30 & 255 \\
\hline 50 & 0 & 0 & 255 & 45 \\
\hline 70 & 0 & 50 & 0 & 255 \\
\hline 80 & 0 & 0 & 0 & 255 \\
\hline 255 & 55 & 0 & 0 & 0 \\
\hline
\end{tabular}

\section{Results and analysis}

The performance of the proposed method is compared with a number of existing filtering techniques. To evaluate the performance, several quantitative measures(criteria) are calculated. The quantitative measures include root-mean square error (RMSE), peak signal to noise ratio (PSNR), image enhancement factor (IEF), and structural similarity (SSIM), which are given in Eqs. 5, 6, 7, 8 respectively.

$$
\begin{gathered}
P S N R\left(\xi_{1}, \xi_{2}\right)=20 \log _{10} \frac{255}{R M S E} \\
\operatorname{RMSE}\left(\xi_{1}, \xi_{2}\right)=\sqrt{\left(\frac{1}{P \times Q} \sum_{p=1}^{P} \sum_{q=1}^{Q}\left|\xi_{1}(p, q)-\xi_{2}(p, q)\right|^{2}\right)} \\
I E F\left(\xi_{1}, \xi_{2}, \xi_{3}\right)=\frac{\sum_{p=1}^{P} \sum_{q=1}^{Q}\left[\xi_{1}(p, q)-\xi_{3}(p, q)\right]^{2}}{\sum_{p=1}^{P} \sum_{q=1}^{Q}\left[\xi_{1}(p, q)-\xi_{2}(p, q)\right]^{2}} \\
S S I M=\frac{\left(2 \omega_{\xi_{1}} \omega_{\xi_{2}}+k_{1}\right)+\left(2 \nu_{\xi_{1} \xi_{2}}+k_{2}\right)}{\left(\omega_{\xi_{1}}^{2}+\omega_{\xi_{2}}^{2}+k_{1}\right)+\left(\nu_{\xi_{1}}^{2}+\nu_{\xi_{2}}^{2}+k_{2}\right)}
\end{gathered}
$$

where $\xi_{1}(p, q), \xi_{2}(p, q), \xi_{3}(p, q)$ are pixels of the original image $\xi_{1}$, restored image $\xi_{2}$ and corrupted image $\xi_{3}$ of size $P \times Q$ respectively. $\omega_{\xi_{1}}, \omega_{\xi_{2}}$ are the average intensities and $\nu_{\xi_{1}}, \nu_{\xi_{2}}$ are standard deviations of image $\xi_{1}, \xi_{2}$ respectively. $\nu_{\xi_{1} \xi_{2}}$ is the covariance of the two images. $k_{1}$ and $k_{2}$ are constants value and are set to $(0.01 \times 255)^{2}$ and $(0.03 \times 255)^{2}$. 
The estimation of salt and pepper noise suppression is performed using 8-bit gray scale images, Airplane, Lena, Bridge, Boats, Mandrill, Peppers, Zelda, Barbara, Goldhill, Flower, Lighthouse, Girl, Moon, and Parrots with different resolutions $512 \times 512,256 \times 256,787 \times 576,720 \times 576,512 \times$ 768. The proposed method is compared with classic filters such as SMF [1] and other recent state-of-the-art techniques like FIDT [6], AFSMF [15], UTMF [16], IDMF [2], DTMF [19] and ERA [20].

Table. 1, Table. 2, Table. 3, and Table. 4 show the restoration results based on comparisons of PSNR(dB), RMSE and IEF and SSIM values obtained by the above existing method and the proposed algorithm for an Airplane image corrupted with the noise density 10\%, 20\%, 30\%, 40\%, 50\%, 60\%, 70\%, 80\%, 90\%, and 99\%. From these table, upto 50\% of noise density all method gives good results to remove impulse noise and preserve more details. This proposed method can successfully remove the noise even at high density noise ratios, and preserve fine edges in the process of denoising.

Table 1: RMSE value of the various noise density $10 \%$ to $99 \%$ using SMF, FIDT, UTMF, AFSMF, IDMF, DTMF ERA and Proposed method for Airplane image.

\begin{tabular}{|c|c|c|c|c|c|c|c|c|c|c|c|}
\hline Noise density (\%) & $10 \%$ & $20 \%$ & $30 \%$ & $40 \%$ & $50 \%$ & $60 \%$ & $70 \%$ & $80 \%$ & $90 \%$ & $95 \%$ & $99 \%$ \\
\hline SMF & 7.33 & 9.00 & 11.85 & 14.69 & 20.15 & 28.20 & 42.91 & 63.96 & 99.88 & 123.11 & 140.64 \\
\hline FIDT & 2.98 & 4.57 & 7.02 & 9.92 & 15.57 & 23.00 & 35.84 & 53.49 & 85.26 & 109.28 & 137.26 \\
\hline UTMF & 2.91 & 4.46 & 6.56 & 8.28 & 10.84 & 14.20 & 19.40 & 30.91 & 56.57 & 75.49 & 97.41 \\
\hline AFSMF & 3.04 & 4.62 & 6.19 & 7.69 & 9.51 & 11.99 & 15.61 & 19.62 & 27.97 & 46.77 & 102.23 \\
\hline IDMF & 6.70 & 7.34 & 8.70 & 9.92 & 11.85 & 13.90 & 19.43 & 27.92 & 44.29 & 55.52 & 77.86 \\
\hline DTMF & 2.97 & 4.59 & 6.00 & 7.27 & 8.64 & 10.18 & 12.12 & 14.74 & 20.91 & 28.16 & 44.48 \\
\hline ERA & 8.01 & 8.58 & 10.48 & 10.68 & 10.99 & 11.66 & 13.35 & 14.59 & 17.77 & 20.94 & 61.36 \\
\hline Proposed method & 2.62 & 3.91 & 5.04 & 6.13 & 7.19 & 8.51 & 10.26 & 12.27 & 16.23 & 20.63 & 32.92 \\
\hline
\end{tabular}

Table 2: PSNR value of the various noise density $10 \%$ to $99 \%$ using SMF, FIDT, UTMF, AFSMF, IDMF, DTMF ERA and Proposed method for Airplane image.

\begin{tabular}{|c|c|c|c|c|c|c|c|c|c|c|c|}
\hline Noise density (\%) & $10 \%$ & $20 \%$ & $30 \%$ & $40 \%$ & $50 \%$ & $60 \%$ & $70 \%$ & $80 \%$ & $90 \%$ & $95 \%$ & $99 \%$ \\
\hline SMF & 30.82 & 29.04 & 26.65 & 24.78 & 22.04 & 19.12 & 15.47 & 12.01 & 8.14 & 6.32 & 5.16 \\
\hline FIDT & 38.64 & 34.92 & 31.19 & 28.19 & 24.28 & 20.89 & 17.04 & 13.56 & 9.51 & 7.35 & 5.37 \\
\hline UTMF & 38.82 & 35.13 & 31.79 & 29.76 & 27.42 & 25.08 & 22.37 & 18.32 & 13.07 & 10.57 & 8.35 \\
\hline AFSMF & 38.44 & 34.82 & 32.28 & 30.40 & 28.56 & 26.55 & 24.25 & 22.27 & 19.19 & 14.77 & 7.93 \\
\hline IDMF & 31.60 & 30.80 & 29.33 & 28.19 & 26.65 & 25.26 & 22.35 & 19.21 & 15.20 & 13.24 & 10.30 \\
\hline DTMF & 38.66 & 34.88 & 32.55 & 30.89 & 29.39 & 27.96 & 26.45 & 24.75 & 21.72 & 19.13 & 15.16 \\
\hline ERA & 30.05 & 29.45 & 27.71 & 27.55 & 27.31 & 26.78 & 25.61 & 24.84 & 23.13 & 21.70 & 12.37 \\
\hline Proposed method & 39.76 & 36.27 & 34.07 & 32.37 & 30.98 & 29.52 & 27.90 & 26.35 & 23.92 & 21.83 & 17.77 \\
\hline
\end{tabular}

Table 3: IEF value of the various noise density $10 \%$ to $99 \%$ using SMF, FIDF, UTMF, AFSMF, IDMF, DTMF ERA and Proposed method for Airplane image.

\begin{tabular}{|c|c|c|c|c|c|c|c|c|c|c|c|}
\hline Noise density $(\%)$ & $10 \%$ & $20 \%$ & $30 \%$ & $40 \%$ & $50 \%$ & $60 \%$ & $70 \%$ & $80 \%$ & $90 \%$ & $95 \%$ & $99 \%$ \\
\hline SMF & 39.2227 & 52.0220 & 45.0219 & 39.0067 & 25.9592 & 15.9179 & 8.0366 & 4.1279 & 1.9082 & 1.3236 & 1.0544 \\
\hline FIDT & 237.5110 & 201.6395 & 128.0174 & 85.4223 & 43.4505 & 23.9387 & 11.5200 & 5.9024 & 2.6188 & 1.6798 & 1.1069 \\
\hline UTMF & 247.6817 & 211.3212 & 146.9016 & 122.6140 & 89.5723 & 62.7516 & 39.2956 & 17.6739 & 5.9487 & 3.5200 & 2.1977 \\
\hline AFSMF & 226.8228 & 197.0394 & 164.7058 & 142.1571 & 116.4226 & 88.0235 & 60.6538 & 43.8631 & 43.8631 & 24.3337 & 9.2746 \\
\hline IDMF & 46.9830 & 78.0337 & 83.4129 & 85.4539 & 75.0333 & 65.3979 & 39.1682 & 21.6632 & 9.7029 & 6.5072 & 3.4398 \\
\hline DTMF & 238.7518 & 199.4038 & 175.1922 & 159.0205 & 141.0029 & 122.0603 & 100.6407 & 77.6854 & 43.5323 & 25.2845 & 10.5402 \\
\hline ERA & 32.8190 & 57.1811 & 57.4877 & 73.7596 & 87.2828 & 93.0120 & 82.9751 & 79.2974 & 60.2852 & 45.7326 & 5.5384 \\
\hline Proposed method & 307.1414 & 275.2047 & 248.6116 & 223.9391 & 203.4162 & 174.5540 & 140.3543 & 112.1143 & 72.2631 & 47.0942 & 19.2346 \\
\hline
\end{tabular}

Figure. 1- 4 show the denoised image of Airplane using the methods SMF, FIDT, AFSMF, UTMF, IDMF, DTMF, ERA and the proposed method with a noise ratio of $20 \%, 40 \%, 70 \%$ and $90 \%$. Also, Figure. 5- 8 show the output denoised image of Boat image using the above methods. From the above results, SMF gives poor performance in all noise densities. FIDT, UTMF, and IDMF filters have considerable improvements over SMF. AFSMF, DTMF and ERA performs better than the FIDT, UTMF, and IDMF filters with respect 
Table 4: SSIM value of the various noise density 10\% to 99\% using SMF, FIDT, UTMF, AFSMF, IDMF, DTMF ERA and Proposed method for Airplane image.

\begin{tabular}{|c|c|c|c|c|c|c|c|c|c|c|c|}
\hline Noise density $(\%)$ & $10 \%$ & $20 \%$ & $30 \%$ & $40 \%$ & $50 \%$ & $60 \%$ & $70 \%$ & $80 \%$ & $90 \%$ & $95 \%$ & $99 \%$ \\
\hline SMF & 0.9926 & 0.9899 & 0.9854 & 0.9782 & 0.9632 & 0.9336 & 0.8593 & 0.7346 & 0.4973 & 0.3675 & 0.2854 \\
\hline FIDT & 0.9990 & 0.9977 & 0.9950 & 0.9902 & 0.9791 & 0.9555 & 0.9005 & 0.8057 & 0.5892 & 0.4445 & 0.2992 \\
\hline UTMF & 0.9990 & 0.9978 & 0.9957 & 0.9930 & 0.9889 & 0.9816 & 0.9689 & 0.9395 & 0.8002 & 0.6380 & 0.4353 \\
\hline AFSMF & 0.9991 & 0.9980 & 0.9959 & 0.9943 & 0.9918 & 0.9888 & 0.9843 & 0.9782 & 0.9782 & 0.9519 & 0.8265 \\
\hline IDMF & 0.9953 & 0.9944 & 0.9923 & 0.9901 & 0.9865 & 0.9809 & 0.9702 & 0.9502 & 0.8863 & 0.8180 & 0.6301 \\
\hline DTMF & 0.9991 & 0.9980 & 0.9960 & 0.9944 & 0.9920 & 0.9890 & 0.9834 & 0.9762 & 0.9572 & 0.9290 & 0.8317 \\
\hline ERA & 0.9871 & 0.9866 & 0.9783 & 0.9784 & 0.9776 & 0.9768 & 0.9684 & 0.9657 & 0.9490 & 0.9361 & 0.7378 \\
\hline Proposed method & 0.9991 & 0.9981 & 0.9969 & 0.9956 & 0.9937 & 0.9913 & 0.9878 & 0.9828 & 0.9710 & 0.9577 & 0.8589 \\
\hline
\end{tabular}

quality measurement parameters such as RMSE, PSNR, IEF, and SSIM. Compared to such existing filters, the proposed method has significant improvement. Figure. 9 shows the local features (RMSE, PSNR, IEF and SSIM) for Boat image using existing filters; SMF, FIDT, AFSMF, UTMF, IDMF, DTMF, ERA and proposed method with noise density $10 \%$ to $90 \%$.

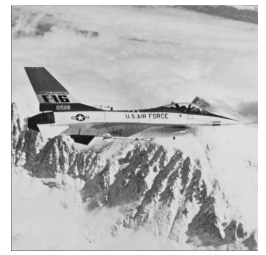

(a)

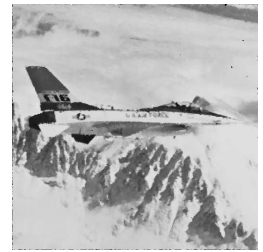

(c)

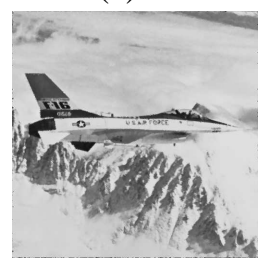

(g)

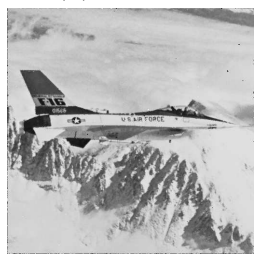

(d)

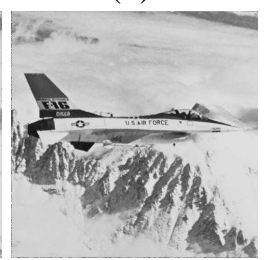

(h)

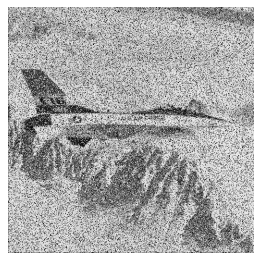

(b)

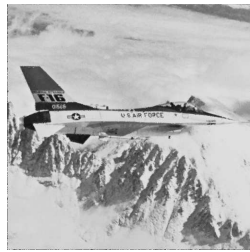

(e)

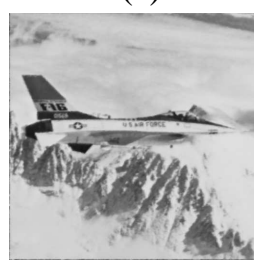

(i)

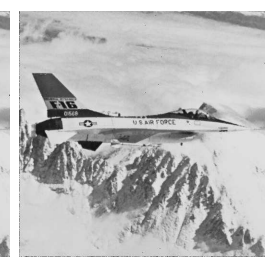

(f)

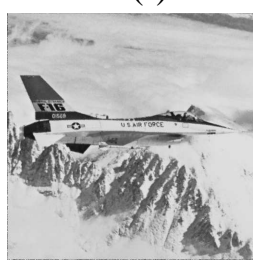

(j)

Figure 1: Output results for Airplane image corrupted with 30\% salt-and-pepper noise density. (a) Original, (b) noisy, (c) SMF, (d) FIDT, (e) UTMF, (f) AFSMF, (g) IDMF, (h) DTMF, (i) ERA and (j) Proposed method.

Figure. 10 - 11 show the denoised image for Goldhill image with corrupted 20\%, 40\% noise ratio of all the above methods, respectively. Compared with all the results, theoretically and visually our proposed method gives the better results than the above existing noise removal algorithms.

Figure. 12 - 13 show the denoised image for Lena, Mandrill, Goldhill and Zelda images with 80\% and 90\% noise ratio using SMF, FIDT, AFSMF, UTMF, IDMF, DTMF, ERA and proposed method. Figure. 14 shows the local features average PSNR and average SSIM of traditional 12 images. From experimental results, our proposed method gives the improvement results than the above existing noise removal techniques. 


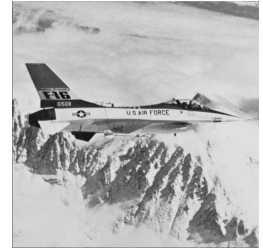

(a)

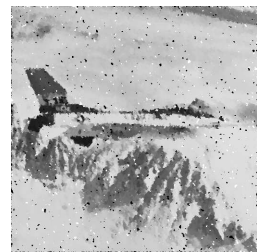

(c)

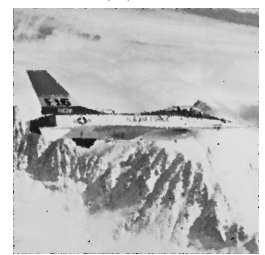

(g)

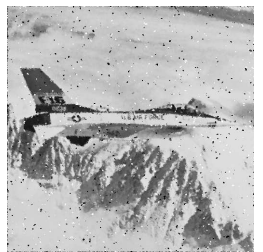

(d)

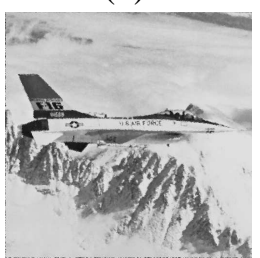

(h)

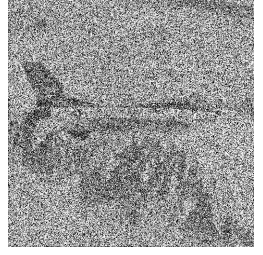

(b)

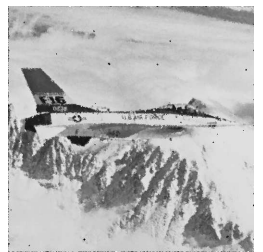

(e)

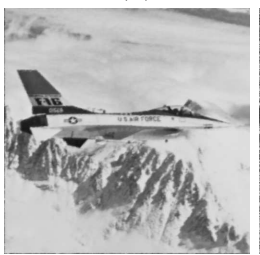

(i)

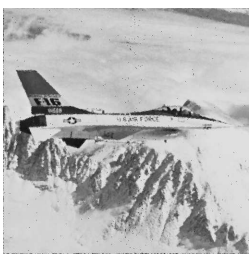

(f)

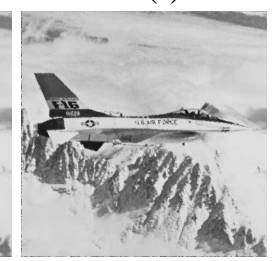

(j)

Figure 2: Output results for Airplane image corrupted with 60\% salt-and-pepper noise density. (a) Original, (b) noisy, (c) SMF, (d) FIDT, (e) UTMF, (f) AFSMF, (g) IDMF, (h) DTMF, (i) ERA and (j) Proposed method.

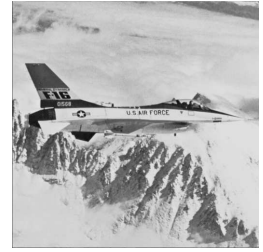

(a)

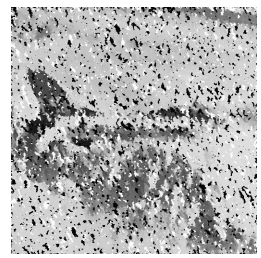

(c)

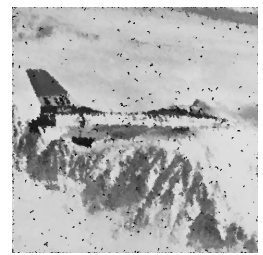

(g)

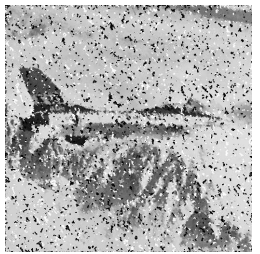

(d)

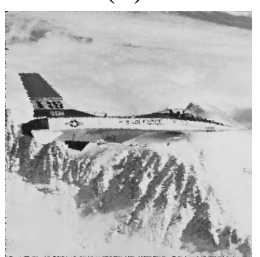

(h)

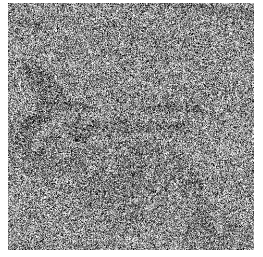

(b)

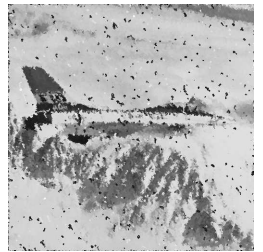

(e)

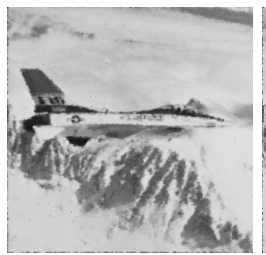

(i)

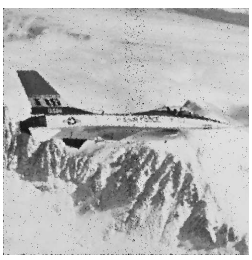

(f)

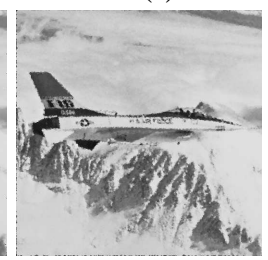

(j)

Figure 3: Output results for Airplane image corrupted with 80\% salt-and-pepper noise density. (a) Original, (b) noisy, (c) SMF, (d) FIDT, (e) UTMF, (f) AFSMF, (g) IDMF, (h) DTMF, (i) ERA and (j) Proposed method. 


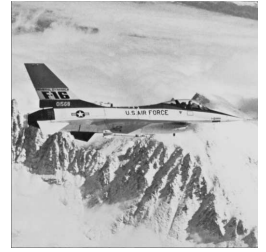

(a)

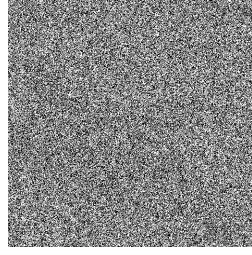

(b)

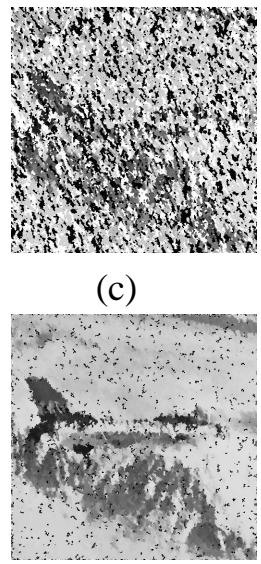

(g)

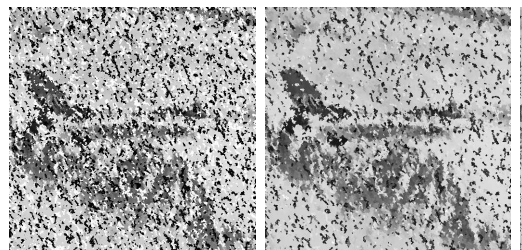

(d)

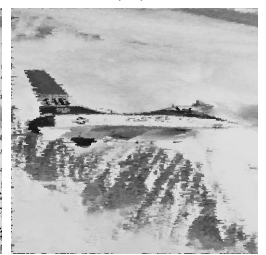

(h) (e)

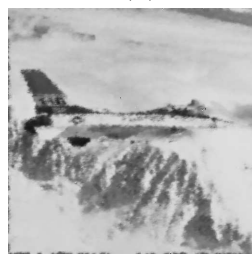

(i)

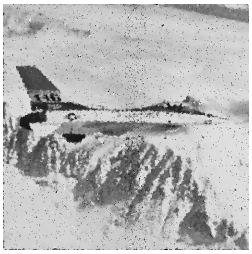

(f)

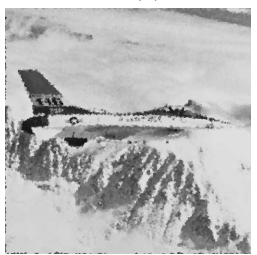

(j)

Figure 4: Output results for Airplane image corrupted with 90\% salt-and-pepper noise density. (a) Original, (b) noisy, (c) SMF, (d) FIDT, (e) UTMF, (f) AFSMF, (g) IDMF, (h) DTMF, (i) ERA and (j) Proposed method.

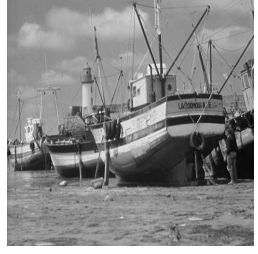

(a)

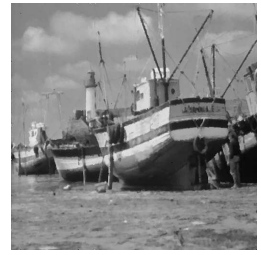

(c)

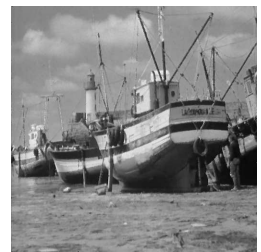

(g)

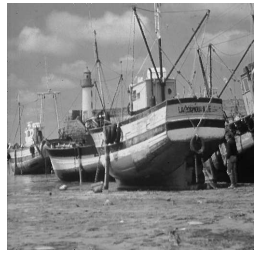

(d)

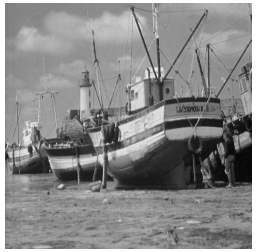

(h)

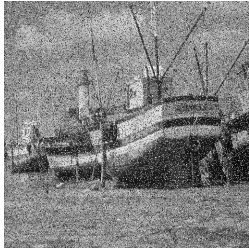

(b)

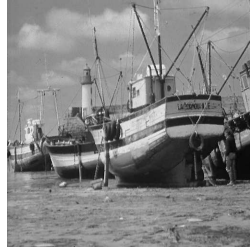

(e)

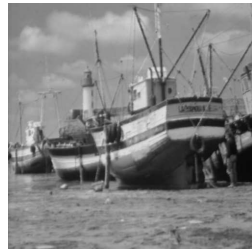

(i)

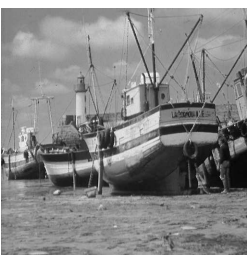

(f)

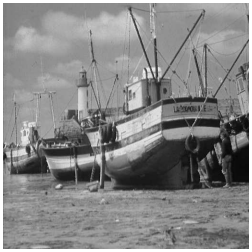

(j)

Figure 5: Output results for Boat image corrupted with 20\% salt-and-pepper noise density. (a) Original, (b) noisy, (c) SMF, (d)FIDT, (e)UTMF, (f) AFSMF, (g) IDMF, (h)DTMF, (i) ERA and (j) Proposed method. 


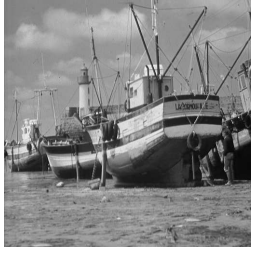

(a)

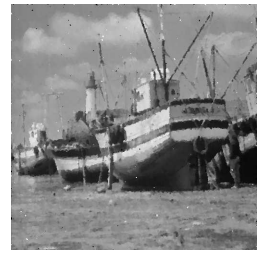

(c)

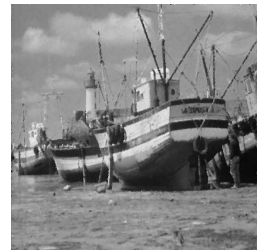

(g)

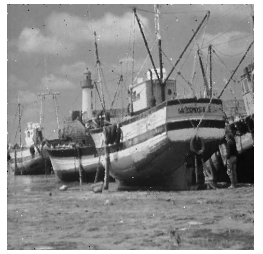

(d)

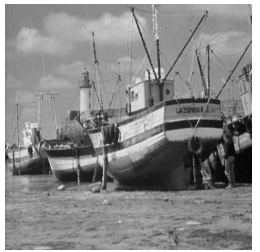

(h)

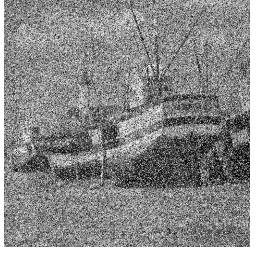

(b)

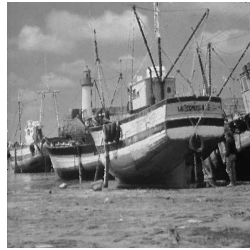

(e)

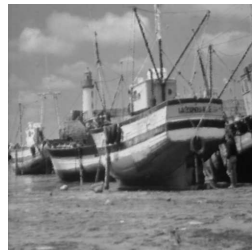

(i)

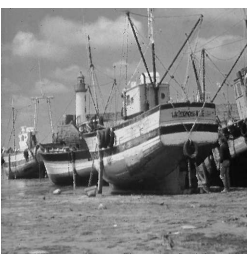

(f)

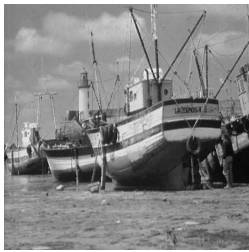

(j)

Figure 6: Output results for Boat image corrupted with 40\% salt-and-pepper noise density. (a) Original, (b) noisy, (c) SMF, (d) FIDT, (e) UTMF, (f) AFSMF, (g) IDMF, (h) DTMF, (i) ERA and (j) Proposed method.

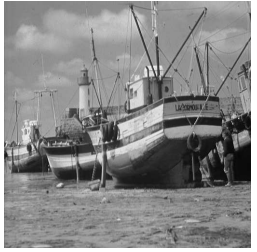

(a)

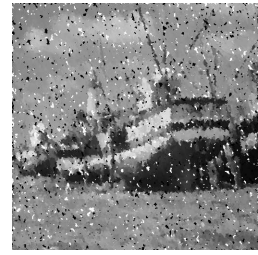

(c)

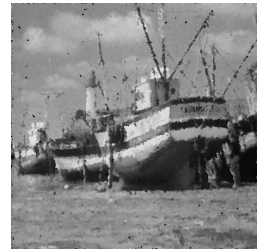

(g)

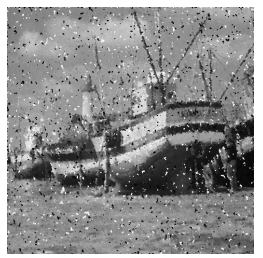

(d)

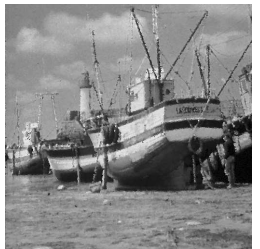

(h)

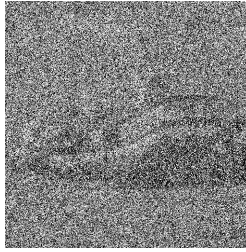

(b)

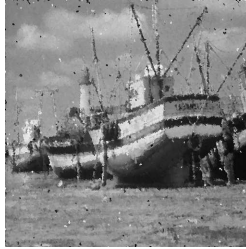

(e)

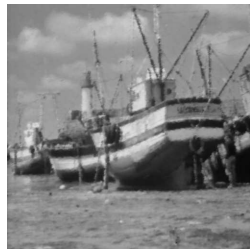

(i)

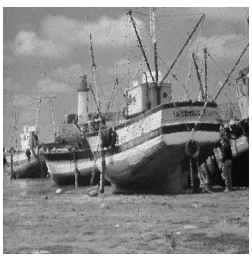

(f)

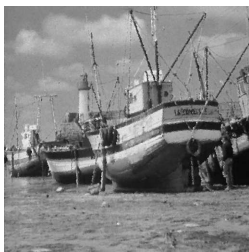

(j)

Figure 7: Output results for Boat image corrupted with 70\% salt-and-pepper noise density. (a) Original, (b) noisy, (c) SMF, (d) FIDT, (e) UTMF, (f) AFSMF, (g) IDMF, (h) DTMF, (i) ERA and (j) Proposed method. 


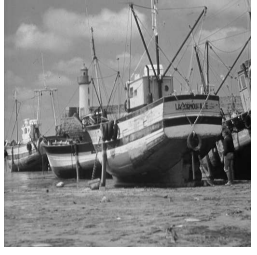

(a)

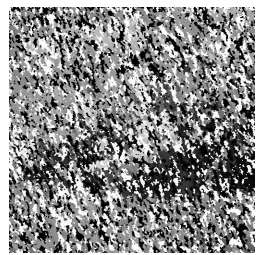

(c)

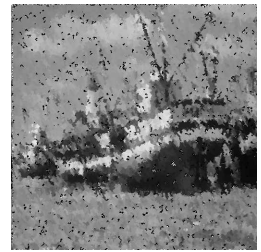

(g)

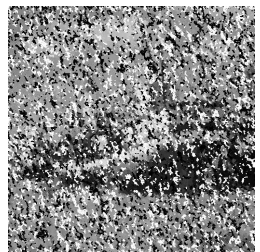

(d)

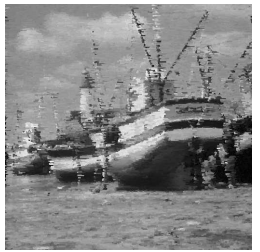

(h)

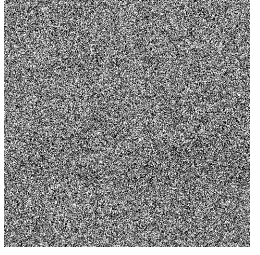

(b)

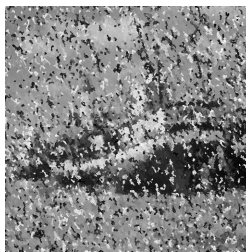

(e)

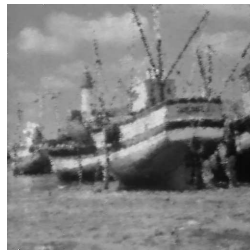

(i)

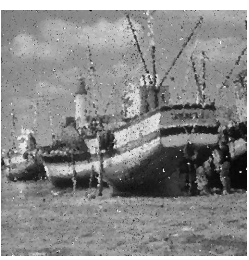

(f)

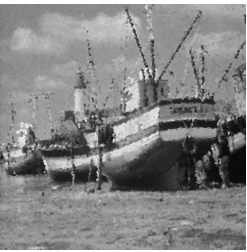

(j)

Figure 8: Output results for Boat image corrupted with 90\% salt-and-pepper noise density. (a) Original, (b) noisy, (c) SMF, (d) FIDT, (e) UTMF, (f) AFSMF, (g) IDMF, (h) DTMF, (i) ERA and (j) Proposed method.

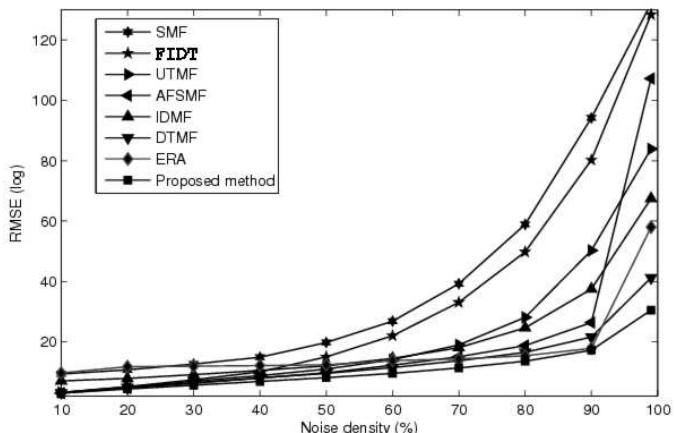

(a)

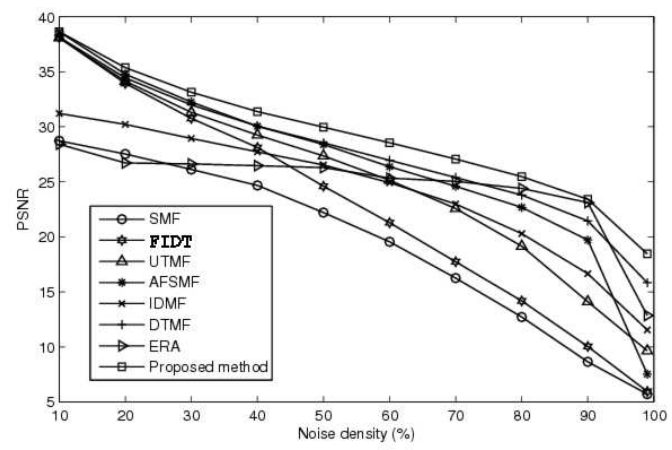

(c)

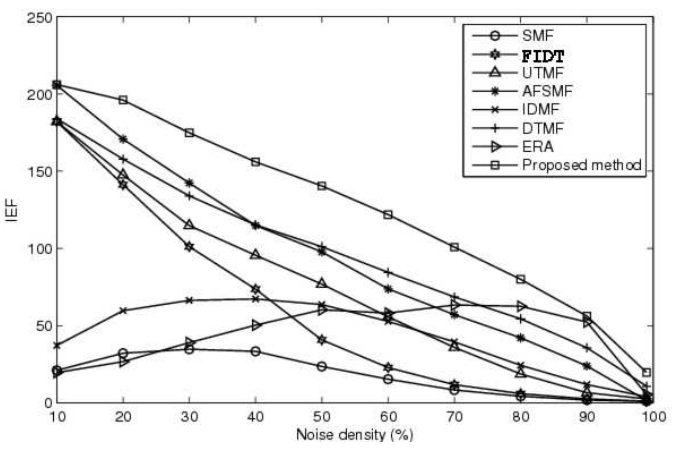

(b)

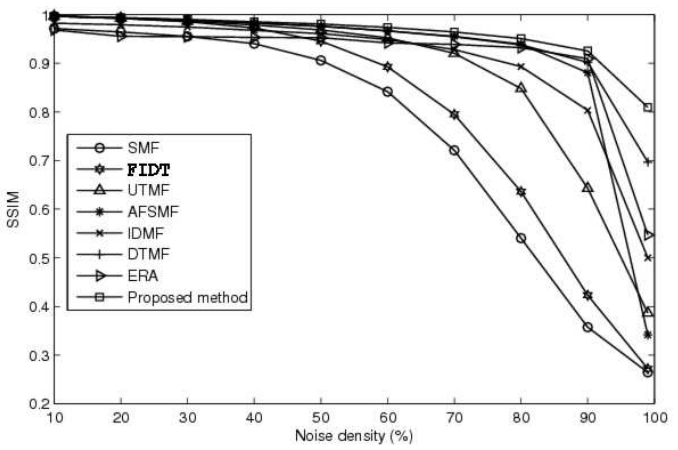

(d)

Figure 9: Comparison of Boat images: (a) RMSE (b) IEF (c) PSNR and (d) SSIM. 


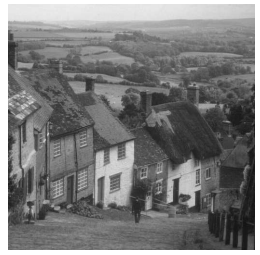

(a)

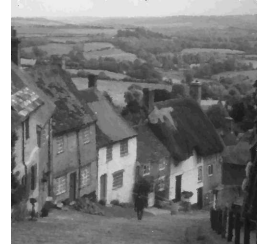

(c)

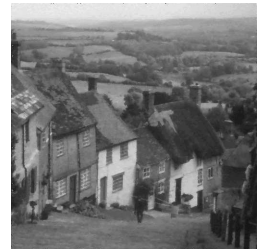

(g)

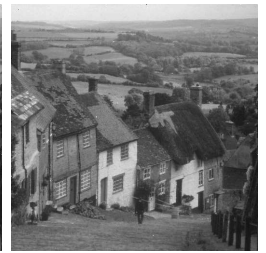

(d)

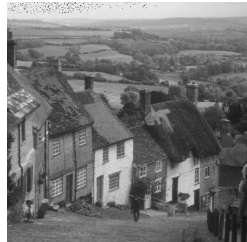

(h)

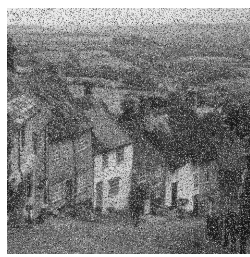

(b)

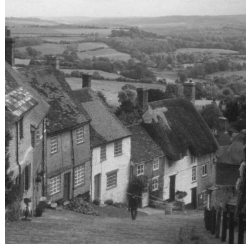

(e)

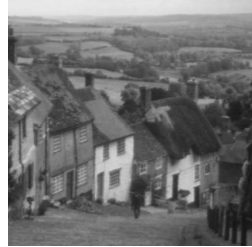

(i)

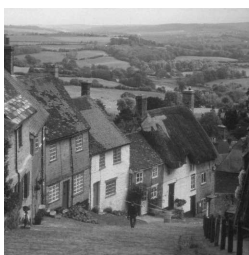

(f)

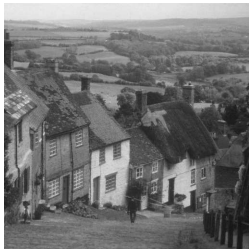

(j)

Figure 10: Output results for Goldhill image corrupted with 20\% salt-and-pepper noise density. (a) Original, (b) noisy, (c) SMF, (d) FIDT, (e) UTMF, (f) AFSMF, (g) IDMF, (h) DTMF, (i) ERA and (j) Proposed method.

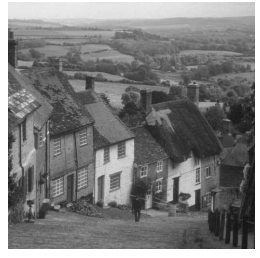

(a)

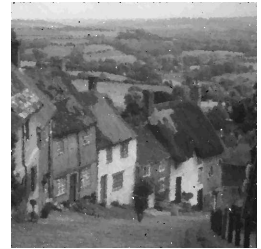

(c)

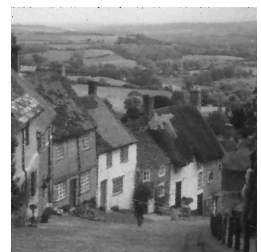

(g)

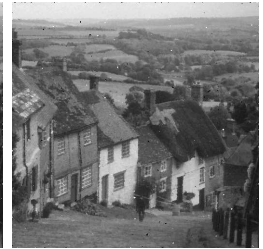

(d)

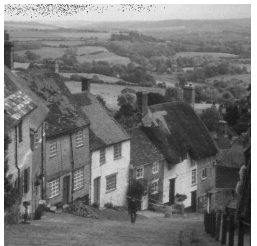

(h)

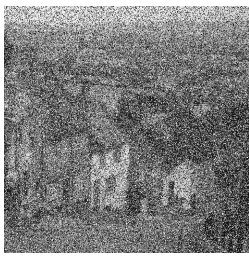

(b)

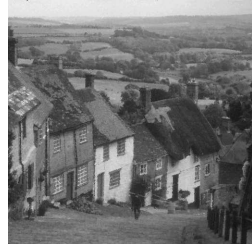

(e)

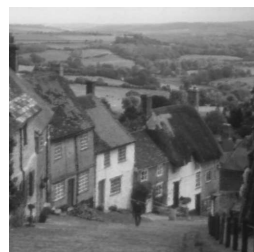

(i)

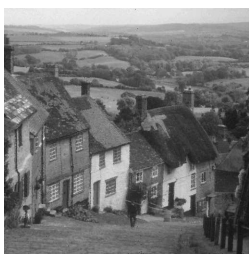

(f)

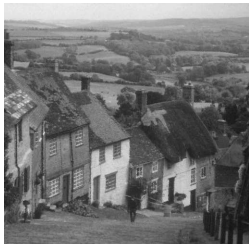

(j)

Figure 11: Output results for Goldhill image corrupted with 40\% salt-and-pepper noise density. (a) Original, (b) noisy, (c) SMF, (d) FIDT, (e) UTMF, (f) AFSMF, (g) IDMF, (h) DTMF, (i) ERA and (j) Proposed method. 
(a)
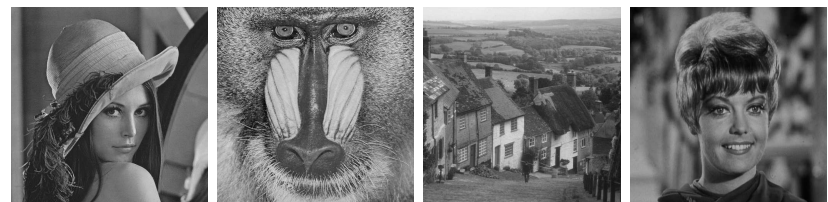

(b)
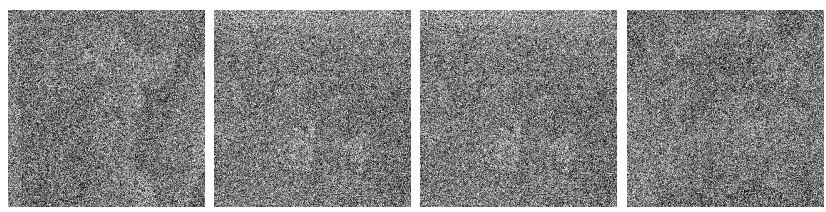

(c)
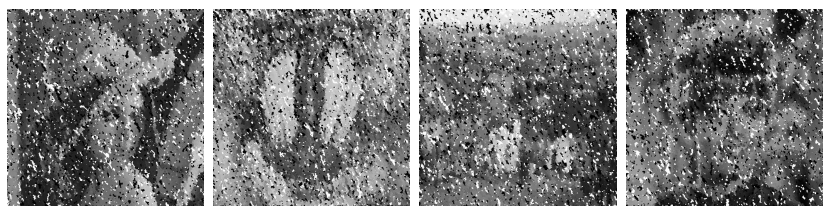

(d)
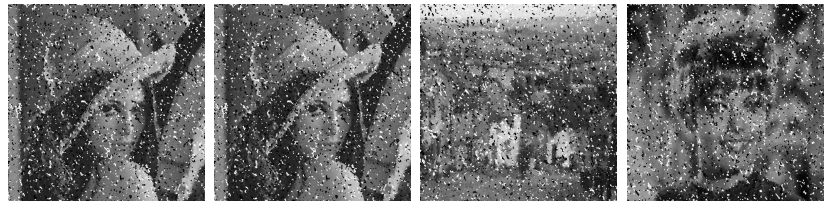

(e)
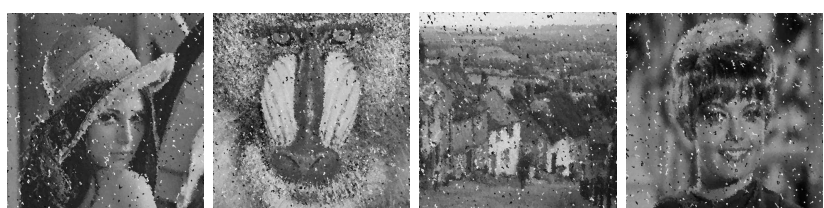

(f)
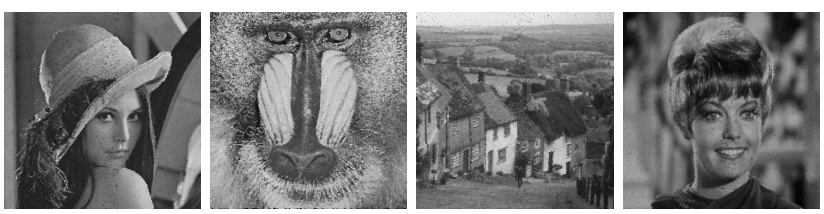

(g)
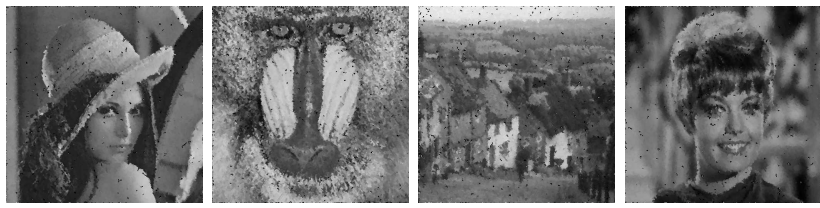

(h)
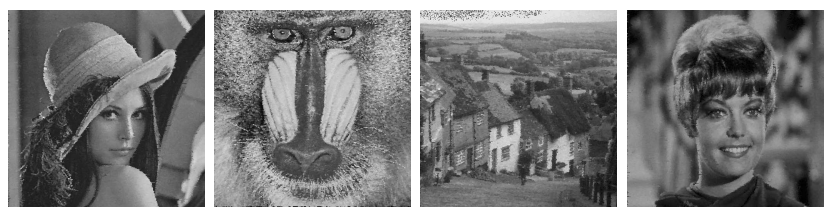

(i)
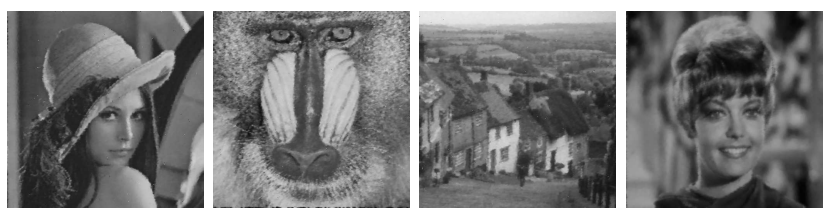

(j)
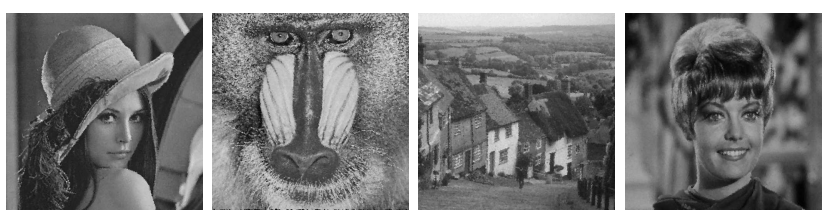

Figure 12: Output results for goldhill image corrupted with $80 \%$ salt-and-pepper noise density. (a) Original, (b) noisy, (c) SMF, (d) FIDT, (e) UTMF, (f) AFSMF, (g) IDMF, (h) DTMF, (i) ERA (j) Proposed method. 
(a)
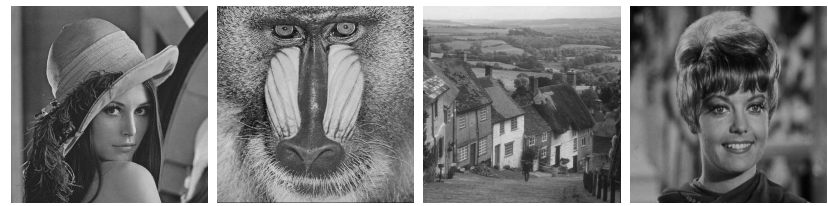

(b)
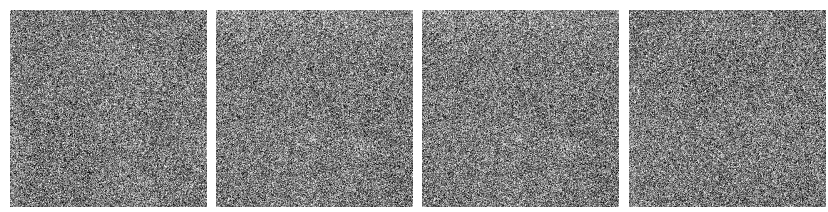

(c)
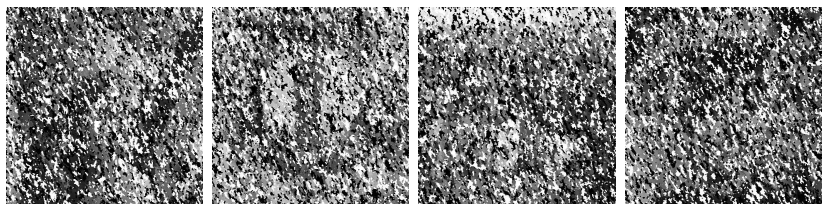

(d)
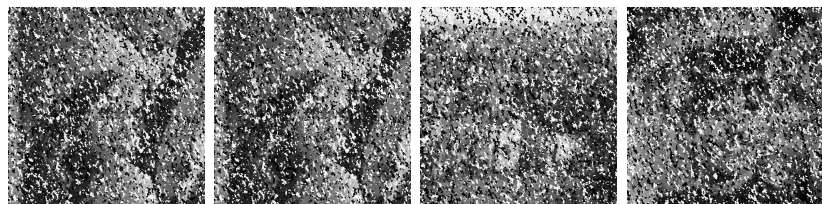

(e)
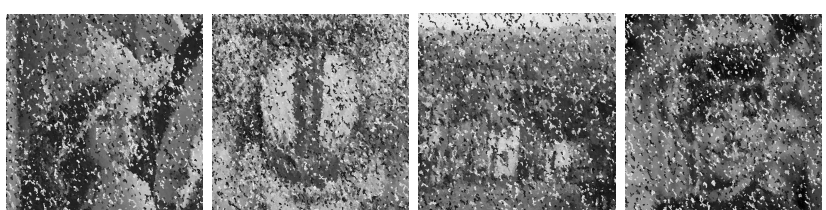

(f)
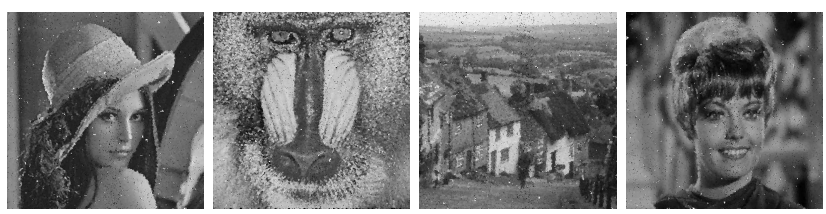

(g)
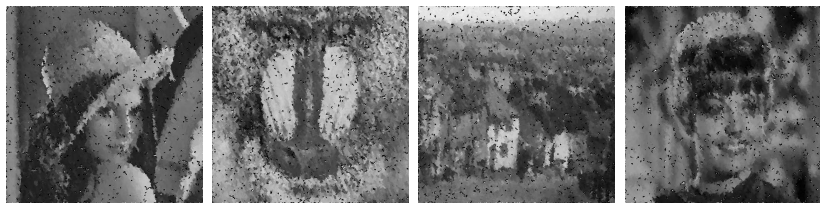

(h)
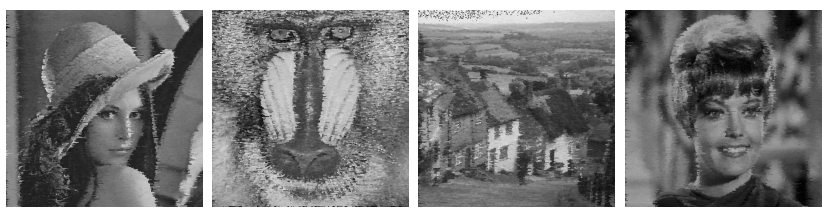

(i)
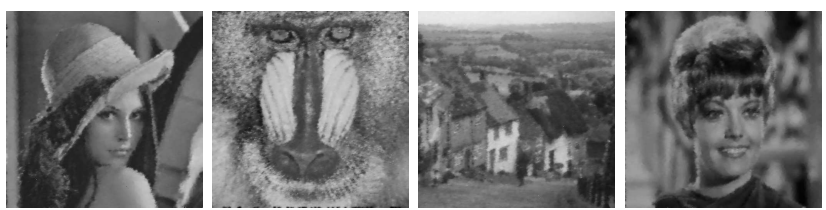

(j)
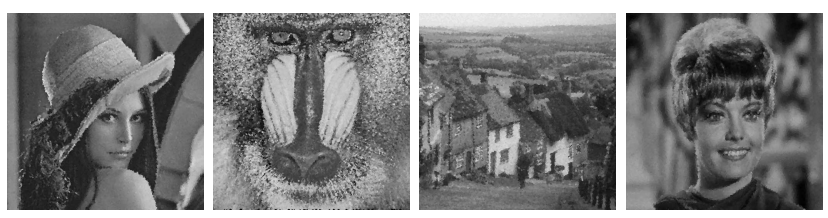

Figure 13: Output results for Goldhill image corrupted with $90 \%$ salt-and-pepper noise density. (a) Original, (b) noisy, (c) SMF, (d) FIDT, (e) UTMF, (f) AFSMF, (g) IDMF, (h) DTMF, (i) ERA and (j) Proposed method. 


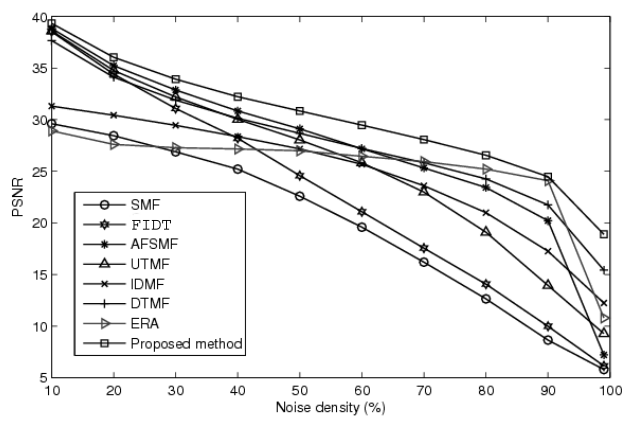

(a)

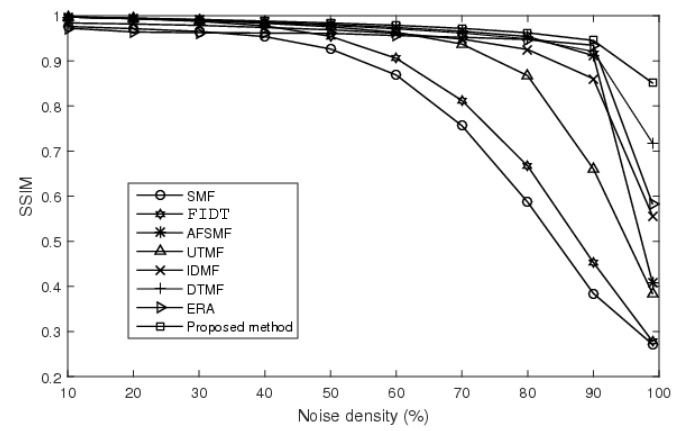

(b)

Figure 14: Comparison of the average (a) PSNR and (b) SSIM value of the twelve Traditional images.

\section{Conclusion}

This paper presents a simple technique to remove salt-and-pepper noise present in different type of images. The technique is based on the mathematical formula of Interquartile range. The proposed technique successfully removes every noisy pixel with suitable intensity value which is calculated based on steps outlined earlier. It has been observed from the experimental results that the proposed method performs better than existing techniques as well as preserve the fine details of the images even if the original image contains high density salt-and-pepper noise. The subjective, as well as objective experimental testing, suggests that the proposed algorithm provides enhanced performance for removing the salt-and-pepper noise overall noise density levels. The proposed technique can be used as pre-processing steps to remove noise in many real-life applications such as medical images and noisy satellite images. With little modification, it can also be applicable to noisy color images.

\section{References}

[1] R. C. Gonzalez, R. E. Woods, Digital Image Processing, Pearson Education, 2002.

[2] V. Bhateja, A. Verma, K. Rastogi, C. Malhotra and S. C. Satapathy, "Performance Improvement of Decision Median Filter for Suppression of Salt and Pepper Noise", Advances in Signal Processing and Intelligent Recognition Systems, 264:287-297, 2014.

[3] S. J. Ko, "Center wighted median filter median filters and their applications to image enhancement", IEEE Transactions on Circuits and Systems, 38(9):984-993, 1991.

[4] T. Chen and H. R. Wu, "Adaptive Impulse Detection Using Center-Weighted Median Filters", IEEE Signal Processing Leters, 8(1):1-3, 2001.

[5] T. Chen, K. K. Ma, and L. H. Chen, "Tri-State Median Filter for Image Denoising", IEEE Transactions on Image Processing, 8(12):1834-1838, 1999.

[6] W. Luo, "Efficient Removal of Impulse Noise from Digital Images", IEEE Transactions on Consumer Electronics, 52(2):523-527, 2006.

[7] J. B. Bednar and T. L. Watt, "Alpha-trimmed means and their relationship to median filters", IEEE Trans. Acoust., Speech, Signal Process., 32(1): 145-153, 1984.

[8] A. C. Bovik, T. S. Huang, and D. C. Munson, "A generalization of median filtering using linear combinations of order statistics”, IEEE Trans. Acoust., Speech, Signal Process., 31(6):1342-1350, 1983. 
[9] P. Y. Chen and C. Y. Lien, "An Efficient Edge-Preserving Algorithm for Removal of Salt-and-Pepper Noise", IEEE Signal Processing Leters., 15():833-836, 2008.

[10] Z. Wang and D. Zhang, "Progressive switching median filter for the removal of impulse noise from highly corrupted images”, IEEE Trans. Circuits Syst. II: Analog Digital Signal Process., 46(1):78-80, 1999.

[11] S. Zhang and M. A. Karim, "A new impulse detector for switching median filters", IEEE Signal Processing Leters., 9(11):360-363, 2002.

[12] K. K. Vin Toh, H. Ibrahim, and M. N. Mahyuddin, "Salt-and-Pepper Noise Detection and Reduction Using Fuzzy Switching Median Filter”, IEEE Transactions on Consumer Electronics, 54(4):1956-1961, 2008.

[13] S. Schulte, M. Nachtegael, V. De Witte, D. Van der Weken, and E. E. Kerre, "A Fuzzy Impulse Noise Detection and Reduction Method", IEEE Transactions on Image Processing, 15(5):1153-1162, 2006.

[14] M.H. Hsieh, F.C. Cheng, M. C. Shie, andS. J. Ruan, "Fast and efficient median filter for removing 1-99\% levels of salt-and-pepper noise in images", Engineering Applications of Artificial Intelligence, 26():13331338, 2012.

[15] K. K. V. Toh and N. A.M. Isa, "Noise adaptive fuzzy switching median filter for salt-and-pepper noise reduction", IEEE Signal Processing Leters, 17(3):281-284, 2010.

[16] S. Esakkirajan, T. Veerakumar, Adabala N. Subramanyam, and C. H. PremChand, "Removal of High Density Salt and Pepper Noise Through Modified Decision Based Unsymmetric Trimmed Median Filter", IEEE Signal Processing Leters, 18(5):287-290, 2011.

[17] S. J. Horng, L. Y. Hsu, T. Li, S. Qiao, X. Gong, H. H. Chou and M. K. Khan "Using Sorted Switching Median Filter to remove high-density impulse noises", J. Vis. Commun. Image R., 24(7):956967, 2013.

[18] V. S. Bhadouria, D. Ghoshal, A. Hasan Siddiqi, "A new approach for high density saturated impulse noise removal using decision-based coupled window median filter", SIViP, 8(1):71-84, 2014.

[19] V. R. Vijaykumara and G. Santhanamarib, "New decision-based trimmed median filter for high-density salt-and-pepper noise removal in images", Journal of Electronic Imaging, 23(3):033011-1-033011-14, 2014.

[20] Guangyu Xu and Yu Lin, "An Efficient Restoration Algorithm for Images Corrupted with Salt and Pepper Noise", Proc of 9th International Congress on Image and Signal Processing, BioMedical Engineering and Informatics, 184-188, 2016. 